\title{
DEMO: Virtual Reality Digital Twin for Floating Production Storage and Offloading (FPSO) Units
}

\author{
Diego Barboza, Wesley de Oliveira, Marco Saraiva \\ SENAI Innovation Institute for Virtual Production Systems \\ FIRJAN SENAI \\ Rio de Janeiro, Brazil \\ \{dcbarboza, wloliveira, maasaraiva\}@ firjan.com.br
}

\author{
Leo Soares \\ Repsol Sinopec Brasil \\ Rio de Janeiro, Brazil \\ leonildes.soares@repsolsinopec.com
}

\begin{abstract}
Summary-Digital Twin is one of the key Industry 4.0 technologies that enable the fourth industrial revolution. With recent advances in tech, the availability of billions of IoT sensors gathering data, and virtual and augmented reality visualization tools, Digital Twins are a great way to provide a unified platform to access this data and interact and visualize them in virtual environments. This work presents an FPSO unit Digital Twin that allows collaborative work and data access in virtual reality.
\end{abstract}

Index Terms-Digital Twin, Virtual Reality, Industry 4.0

\section{INTRODUCTION}

Even though the notion of Digital Twin first appeared in the year 2000, the concept of a twin technology date from NASAs Apollo Program, when they created two identical shuttles: one would go to space, while the other would stay on Earth in order to mirror the conditions during flight [1]. This twin concept provided NASA engineers with real-time data feeds from equipment that were running in space, enabling decision making and execution of certain procedures without the presence of a human being alongside the asset.

NASAs twin concept was a precursor to Digital Twins. Thanks to the evolution of IoT technology and the Internet, it is now possible to retrieve and analyze large amounts of data in real-time, enabling the visualization of past data in reports, current data in live feeds, and predict future data using Artificial Intelligence.

A Digital Twin is a bridge between physical and digital worlds [2]. It is a representation of an existing process, product or service, providing data that reflect real-world present and historical conditions of said asset [3], made possible by sensors that collect data in real-time. This pairing of the virtual and physical worlds allows analysis of data and monitoring of systems to determine problems before they occur, prevent downtime, develop new opportunities and even plan for the future through simulations.

Digital Twins can be applied to a variety of scenarios and markets. Real examples of nowadays include:

- Usage in sports for prediction and analysis, like in Football or Formula 1.

- Creating Digital Twins of patients in a hospital, enabling the simulation of procedures in a body to test different treatment scenarios.
- Modeling the Digital Twin of an entire city to detect energy leaks, improve traffic, safety, and many other factors.

Digital Twin technology is on the rise, with predictions of billions of sensors running worldwide by 2020 [4], making Digital Twin possible for a large amount of connected devices providing data in real-time.

\section{OBJeCtive}

The objective of this project is to create a Digital Twin of a Floating Production Storage and Offloading (FPSO) unit. The development was planned for multiple phases, starting with five critical modules as shown in Fig. 1.

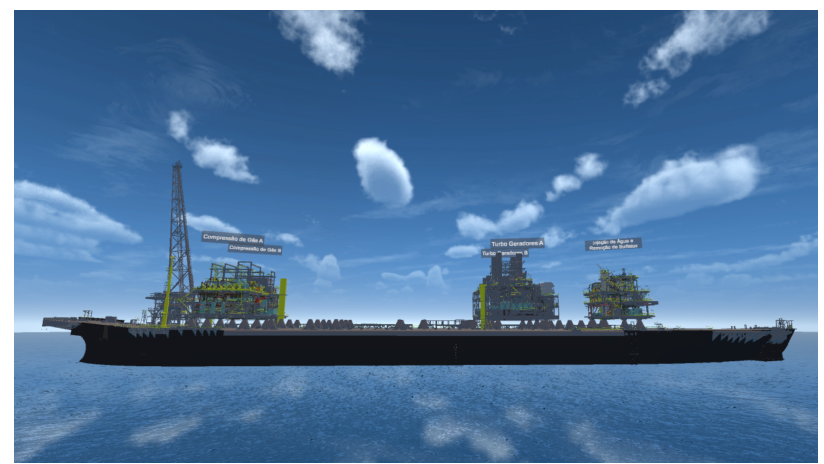

Fig. 1. A panorama view of the Digital Twin and five critical modules loaded.

Fig. 2 presents an overview of the Digital Twins architecture. It works as an interface to assets manuals, reference documents, 3D representation, maintenance orders and historical and real-time sensor data. This data can be used by machine learning algorithms to estimate how each component behaves and trigger alarms when they are out of operating range or by virtual or augmented reality applications to access all of the unified asset data from a single interface, to navigate through the 3D model and to access different data views based on capture data and predictive analysis. This project focuses on the virtual reality tool to access data generated by the Digital Twin and the predictive algorithms, but an augmented reality tool is also on our roadmap.

The user can navigate inside the simulated 3D environment in Virtual Reality (VR) and experience it as being present 


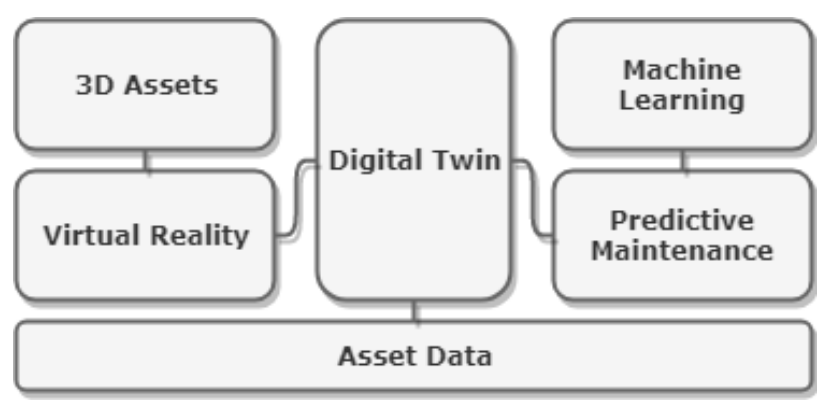

Fig. 2. An overview of the Digital Twins architecture.

inside the Digital Twin, interacting with the assets when needed. This is a collaborative environment where multiple users can access the Digital Twin at the same time and perform tasks or analysis together.

Inside the simulation, the user has several tools to interact with the system: measurement tools for checking distances and sizes of any asset, locomotion tools (teleport and free flight) to facilitate movement inside the VR environment, a pointer tool to interact at a distance with the assets and a search tool to find any asset that has been mapped and sensored by its tag as seen in Fig. 3.

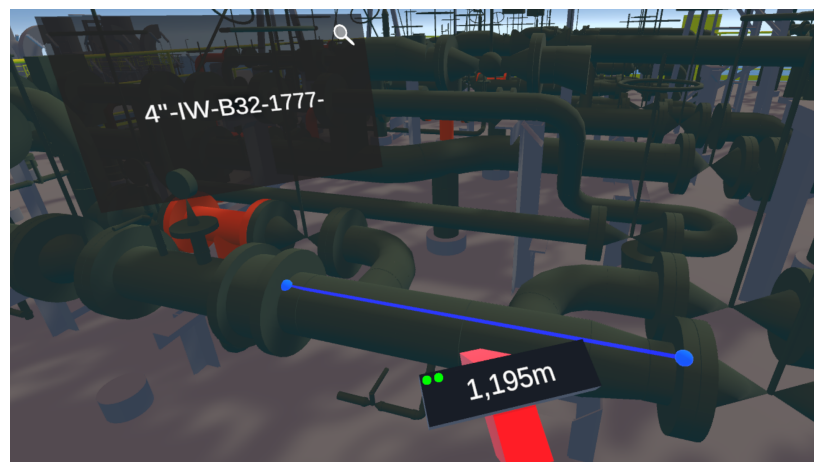

Fig. 3. Example of an asset tag and a measurement tool used on a piping.

The interaction can happen with both the 3D model and its associated data from every tracked asset inside each module. Data generated by sensors of these assets become available in the VR tool through a unified data platform (Asset Data in Fig.2). This data helps to understand the elements that compose the Digital Twin and facilitates the actual operation throughout its lifecycle, allowing adjustments when necessary.

The data has to be accessed through a variety of ways and be able to be parsed and analyzed. It can be viewed directly by interacting in VR with point-and-click interfaces that trigger requests to access data from the unified platform and displays the result to the user in a panel in front of the selected asset. A search can also be done to request an asset by its tag or using a data filter, e.g. all assets with scheduled maintenance this week. These requests are done by a secured external API to access the data in real-time from a data server.

Real-time analysis of data that allows prediction and optimization of the Digital Twin working routine. This prevents accidents [5], reduce maintenance costs [6] and allows a better understanding of the life-cycle of an asset [7].

Finally, the project also encompasses the ability to generate and perform training scenarios within the VR simulation. This allows the employee to perform training of difficult or dangerous tasks without any risks or to prepare for maintenance procedures gathering the information based on all available asset data before performing the actual procedure.

\section{RESUlTS AND Discussion}

This project is still in development but in its current state its already possible to use the Digital Twin as a window to the FPSOs assets in a multi-user collaborative environment. Further development will include training scenarios and procedures for critical operations that users will be able to simulate before going to the field and different visualization modes to quickly retrieve information about the assets current state.

While development for now is mostly focused on virtual reality tools, an augmented reality mode with spatial mapping for on-site data access in also on our roadmap.

\section{CONCLUSION}

We developed a virtual reality tool to access sensor data and documentation for assets of five critical modules in an FPSO unit. It allows the user to navigate the $3 \mathrm{D}$ model and interact with other users to visualize data in multiple ways, perform assessments, training and simulations.

Digital Twin is a concept that can greatly improve productivity and with this tool performance gains and better knowledge retention is expected. This should be tested when the project is deployed and used in a real environment.

Future work include extending the tool to other FPSO modules and use augmented reality with spatial mapping to overlay the Digital Twin data combined with the real assets.

\section{THANKS}

The authors would like to thank Repsol Sinopec Brasil, Petrobras and the Brazilian National Petroleum Agency (ANP) for this research by funding and providing real data and assets.

\section{REFERENCES}

[1] M. Holler, F. Uebernickel, and W. Brenner, "Digital twin concepts in manufacturing industries-a literature review and avenues for further research," 2016.

[2] S. Boschert, C. Heinrich, and R. Rosen, "Next generation digital twin," Proceedings of TMCE, Las Palmas de Gran Canaria, Spain Edited by: Horvath I., Suarez Rivero JP and Hernandez Castellano PM, 2018.

[3] "An introduction to digital twin development," White Paper, Anylogic, 2018.

[4] "Leading the iot, gartner insights on how to lead in a connected world," White Paper, Gartner, 2017.

[5] M. Grieves and J. Vickers, "Digital twin: Mitigating unpredictable, undesirable emergent behavior in complex systems," in Transdisciplinary perspectives on complex systems. Springer, 2017, pp. 85-113.

[6] F. Tao, J. Cheng, Q. Qi, M. Zhang, H. Zhang, and F. Sui, "Digital twindriven product design, manufacturing and service with big data," The International Journal of Advanced Manufacturing Technology, vol. 94, no. 9-12, pp. 3563-3576, 2018.

[7] L. F. C. Durão, S. Haag, R. Anderl, K. Schützer, and E. Zancul, "Digital twin requirements in the context of industry 4.0," in IFIP International Conference on Product Lifecycle Management. Springer, 2018, pp. 204214. 